Gut and Liver, Vol. 11, No. 5, September 2017, pp. 612-619

\title{
Risk Factors for Gastric Tumorigenesis in Underlying Gastric Mucosal Atrophy
}

\author{
Ji Hyun Song ${ }^{1}$, Sang Gyun Kim² , Eun Hyo Jin ${ }^{1}$, Joo Hyun Lim ${ }^{1}$, and Sun Young Yang ${ }^{1}$ \\ ${ }^{1}$ Department of Internal Medicine, Healthcare Research Institute, Seoul National University Hospital Healthcare System Gangnam Center, and \\ ${ }^{2}$ Department of Internal Medicine and Liver Research Institute, Seoul National University College of Medicine, Seoul, Korea
}

See editorial on page 575.

Background/Aims: Atrophic gastritis is considered a premalignant lesion. We aimed to evaluate the risk factors for gastric tumorigenesis in underlying mucosal atrophy. Methods: A total of 10,185 subjects who underwent upper gastrointestinal endoscopy between 2003 and 2004 were enrolled in this retrospective cohort study. Follow-up endoscopy was performed between 2005 and 2014. Atrophic gastritis and intestinal metaplasia were assessed by endoscopy using the Kimura-Takemoto classification. Helicobacter pylori infection was evaluated based on serum immunoglobulin $\mathrm{G}$ antibody levels, the rapid urease test, or the urea breath test. Results: Atrophic gastritis was confirmed in 3,714 patients at baseline; 2,144 patients were followed up for 6.9 years, and 1,138 exhibited increased atrophy. A total of 69 subjects were diagnosed with gastric neoplasm during follow-up (35 adenoma and 34 carcinoma). Age $\geq 55$ years (hazard ratio [HR], 1.234), alcohol consumption (HR, 1.001), and H. pylori infection (HR, 1.580) were associated with increased mucosal atrophy. The risk factors for gastric neoplasm in underlying mucosal atrophy were age $\geq 55$ years (HR, 2.582), alcohol consumption (HR, 1.003), extent of mucosal atrophy (HR, 2.285 in C3-01; HR, 4.187 in 02-03), and intestinal metaplasia (HR, 2.655). Conclusions: Extent of atrophy, intestinal metaplasia, and alcohol consumption are significant risk factors for gastric neoplasm in underlying mucosal atrophy. (Gut Liver 2017;11:612-619)

Key Words: Atrophy; Stomach; Neoplasms; Epidemiology

\section{INTRODUCTION}

Gastric carcinogenesis is believed to develop in a multistep process, progressing from chronic gastritis through atrophic gastritis (AG), intestinal metaplasia (IM), and dysplasia before ending in cancer. ${ }^{1} \mathrm{AG}$ is characterized by the loss of appropriate glands and a reduction in gastric secretory function which results from chronic inflammatory processes in the gastric mucosa. ${ }^{2}$ IM is defined as the replacement of gastric columnar epithelial cells by cells with an intestinal morphology. ${ }^{3}$ The initial stage of gastritis and atrophy is linked to chronic infection with Helicobacter pylori. ${ }^{1}$ Two potential pathways have been proposed. The first one is $H$. pylori-induced chronic inflammation in epithelial cells that progresses into gastric atrophy (the "indirect action") and the other is gastric atrophy resulting from epithelial cells being infected by $H$. pylori bacterial agents such as cytotoxin-associated gene A (the "direct action"). ${ }^{4}$

Gastric cancer is the fourth most common malignancy and the second leading cause of cancer death in the world. ${ }^{5}$ The highest incidence rates are observed in East Asia, South America and Eastern Europe. ${ }^{6}$ A recent cohort study in the United States has reported an increasing trend of noncardiac gastric cancer in the young white population. ${ }^{7}$ Another study in Sweden reported that the prevalence of AG among people aged 35 to 44 had increased over the past 20 years, which implies that the falling trend of gastric cancer might be reversed in the near future. ${ }^{8,9}$ In Korea, the prevalence of endoscopic AG is relatively high: $59.4 \%$ in people older than 60 years. ${ }^{10}$

The Korean National Cancer Screening Program has recommended regular 2-year interval gastric cancer screening by upper endoscopy or upper gastrointestinal barium study for individuals over 40 years of age. ${ }^{11}$ However, there have been no unified surveillance interval guidelines for high-risk patients

Correspondence to: Sang Gyun Kim

Department of Internal Medicine and Liver Research Institute, Seoul National University College of Medicine, 101 Daehak-ro, Jongno-gu, Seoul 03080, Korea

Tel: +82-2-740-8112, Fax: +82-2-743-6701, E-mail: harley1333@hanmail.net

Received on October 4, 2016. Revised on January 4, 2017. Accepted on January 17, 2017. Published online May 23, 2017

pISSN 1976-2283 eISSN 2005-1212 https://doi.org/10.5009/gnl16488

@) This is an Open Access article distributed under the terms of the Creative Commons Attribution Non-Commercial License (http://creativecommons.org/licenses/by-nc/4.0) which permits unrestricted non-commercial use, distribution, and reproduction in any medium, provided the original work is properly cited. 
such as those with AG or IM. A recent Korean study which conducted with an asymptomatic population reported that the rate of early gastric cancer (EGC) was higher in the annual screening group than in the biennial group $(98.6 \%$ vs $80.7 \%, \mathrm{p}<0.01$ ) and endoscopic resection was performed more frequently in the annual group (56.9\% vs 33.3\%, $\mathrm{p}=0.02$ ), thereby suggesting that intensive screening and surveillance might be useful for high-risk subpopulations with epidemiologic risk factors or premalignant lesions. ${ }^{12}$ Another Korean study demonstrated that the proportion of EGC was higher in the vigilant screening group (patients who had undergone endoscopy within the past year) than in the non-vigilant screening group (66.7\% vs $35.5 \%, p=0.047$ ), and that endoscopic resection was performed more frequently in the vigilant group than in the non-vigilant group (26.7\% vs $0 \%, \mathrm{p}=0.028)$. $^{13}$ For these reasons, we have recommended regular surveillance endoscopy every one year for patients with severe AG and/or IM.

While AG is considered as a precancerous lesion, most patients with AG rarely experience gastric cancer. Therefore, stratifying the patients with AG by risk of gastric cancer may be important for the prevention and/or early detection of gastric cancer. Many studies have shown that the risk of gastric cancer was strongly associated with $H$. pylori infection, smoking, high intake of salty and smoked food, and low intake of fruits and vegetables. ${ }^{4}$ However, few studies have been reported to investigate the risk factors for gastric neoplasm in patients with AG. In this study, we aimed to evaluate AG by endoscopy and analyze the risk factors for the aggravation of $A G$ and gastric tumorigenesis in underlying gastric mucosal atrophy.

\section{MATERIALS AND METHODS}

\section{Patients}

Between October 2003 and October 2004, 10,294 subjects underwent upper gastrointestinal endoscopy as part of their health check-up at Seoul National University Hospital Healthcare System Gangnam Center. A total of 109 subjects were excluded from the analysis because of previous gastric resection $(n=62)$ or gastric neoplasm within the first year of surveillance $(n=47 ; 18$ adenoma, 29 carcinoma), resulting in a final cohort of 10,185 subjects whose medical records were reviewed retrospectively. AG was confirmed in 3,714 subjects (36.5\%) by endoscopy, among whom 2,144 were followed up for more than 1 year until December 2014 (Fig. 1).

All subjects were requested to complete a self-administered, structured questionnaire on smoking history, alcohol intake, family history of gastric cancer, and history of $H$. pylori eradication therapy. Alcohol intake was measured by ascertaining the number of drinks per week, which was defined as beer (200 $\mathrm{mL}$ per glass), soju (50 mL per glass), wine (120 mL per glass), or liquor (30 $\mathrm{mL}$ per shot), each being equivalent to approximately $10 \mathrm{~g}$ of alcohol per drink. Heavy drinking was defined as more than 14 drinks per week for men and seven drinks per week for women.

The study protocol was approved by the ethics committee of Seoul National University Hospital (Institutional Review Board number: H-1503-123-658), and was conducted in accordance with the Declaration of Helsinki.

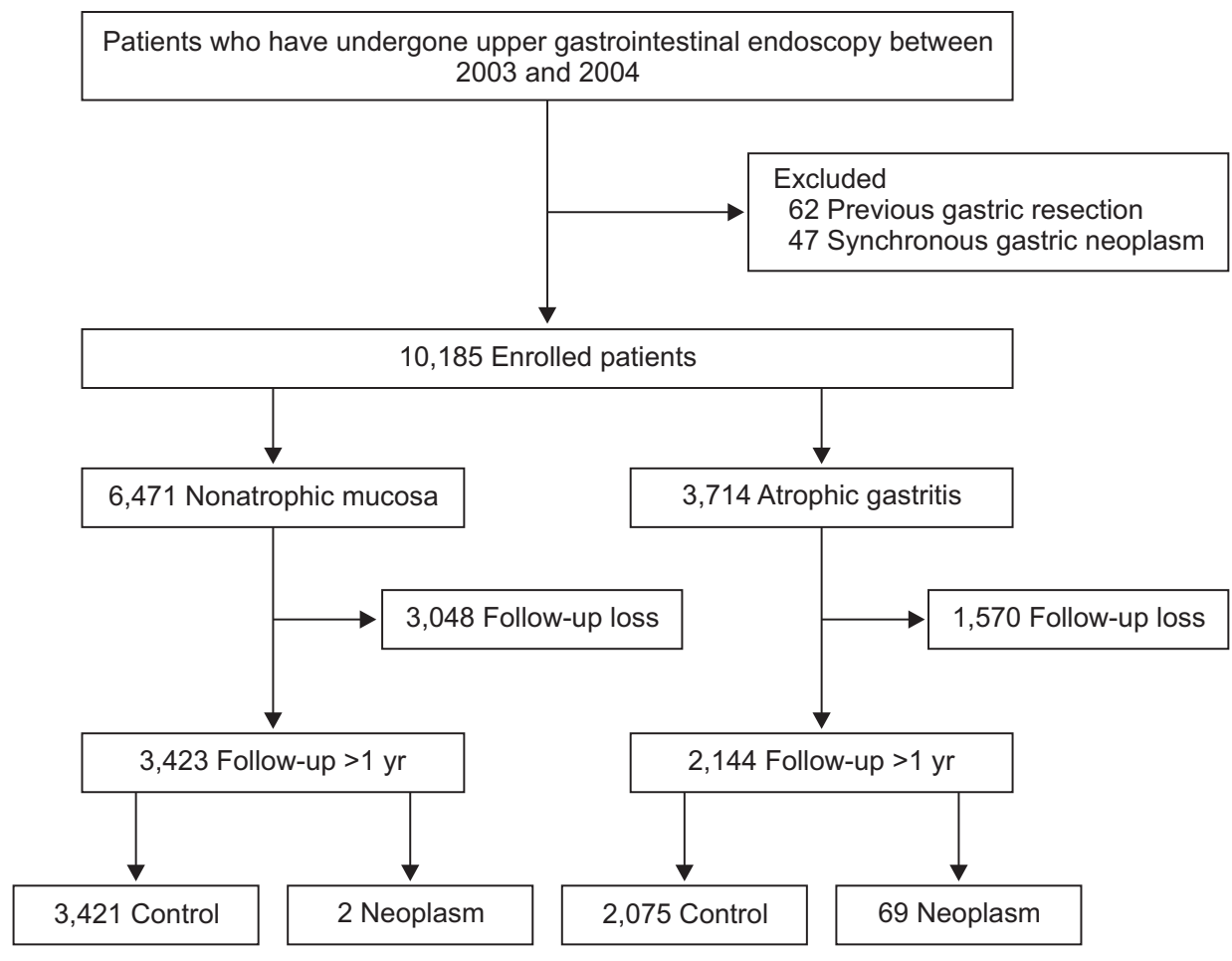

Fig. 1. Flow chart of the study population. 


\section{Endoscopic evaluation}

Endoscopic gastric mucosal atrophy was evaluated according to the Kimura and Takemoto ${ }^{14}$ classification system. Atrophy was classified as either closed type (C1-C3), in which the borders of atrophy did not exceed the cardia on the lesser curvature side of the gastric body, or open type (01-03), in which the borders of atrophy exceeded the cardia into the greater curvature side. ${ }^{14}$ The characteristic features of each type of atrophy were as follows: limited to the antrum in $\mathrm{C} 1$, limited to the gastric angle or the lower body in C2, limited to the upper body in C3, limited to the surroundings of the gastric cardia with maintained folds of the greater curvature in 01 , atrophy in the entire stomach with a lack of folds throughout the greater curvature in 03, and 02 as an intermediate type between 01 and $03 .{ }^{15}$ Endoscopic gastric atrophy was divided into three grades: mild (C1, C2), moderate $(\mathrm{C} 3,01)$, and severe $(02,03)$.

Endoscopic IM was diagnosed when whitish plaque-like elevations were seen in the antrum and/or body. When a diagnosis of IM was not clear or a suspicious neoplastic lesion was seen during endoscopy, an additional biopsy was conducted. Histologic IM was evaluated by the updated Sydney system.

All endoscopic findings were reviewed by four experienced endoscopists (J.H.S., E.H.J., J.H.L., and S.Y.Y.). Several meetings were held to improve the accuracy of diagnosis and minimize interobserver variation by extensively reviewing multiple cases with AG. Interobserver agreements on atrophy grades were assessed by Fleiss' kappa coefficient and were initially not good $(\kappa=0.36, p<0.001)$ but improved substantially later $(\kappa=0.62$, $\mathrm{p}<0.001)$.

\section{Follow-up and management}

Asymptomatic individuals over 40 years of age were recommended to receive upper gastrointestinal endoscopy to screen for gastric cancer every 2 years. Individuals with AG and/or IM during initial screening endoscopy were recommended to receive annual endoscopic examination in our institute. Aggravated AG was defined as when the grade of mucosal atrophy was aggravated (from mild to moderate or severe, and from moderate to severe) or when IM developed by the time of follow-up endoscopy. When gastric neoplasms were diagnosed during follow-up endoscopy, adenoma with low- or high-grade dysplasia was recommended to be removed by endoscopic resection and was then followed up annually. Patients with adenocarcinoma were recommended to be treated by surgical or endoscopic resection according to the indication.

\section{H. pylori status evaluation}

Serum $H$. pylori immunoglobulin G (IgG) antibody test was routinely performed using a commercially available enzymelinked immunosorbent assay (H. pylori-EIA-Well; Radim, Rome, Italy) on the same day of endoscopy. According to the manu- facturer's instructions, an IgG value $>30 \mathrm{U} / \mathrm{mL}$ was considered to be reactive for $H$. pylori, 15 to $30 \mathrm{U} / \mathrm{mL}$ was considered to be weakly reactive, and $<15 \mathrm{U} / \mathrm{mL}$ was considered to be nonreactive. Reactive and weakly reactive were defined as a positive result.

In the presence of peptic ulcer disease, biopsy specimens from the lesser curvature of the antrum and/or body were obtained for rapid urease test (CLO test ${ }^{\circledR}$; Delta West, Bentley, Australia). After $H$. pylori eradication therapy, the ${ }^{13} \mathrm{C}$-urea breath test $(\mathrm{He}-$ liView ${ }^{\circledR}$; Medichems, Seoul, Korea) was used to confirm the current $H$. pylori status.

The subject was considered as H. pylori-positive if any one of

Table 1. Baseline Characteristics of the Study Population $(n=10,185)$

\begin{tabular}{|c|c|}
\hline Characteristic & Value \\
\hline Age, yr & $47.81 \pm 11.22$ \\
\hline$<55$ & $7,339(72.1)$ \\
\hline$\geq 55$ & 2,846 (27.9) \\
\hline Male sex & $5,581(54.8)$ \\
\hline \multicolumn{2}{|l|}{ Smoking* } \\
\hline Nonsmoker & $1,242(45.3)$ \\
\hline Ex-smoker & $1,072(39.1)$ \\
\hline Current smoker & $425(15.5)$ \\
\hline Smoking, pack-year ${ }^{\dagger}$ & $11.25 \pm 16.59$ \\
\hline \multicolumn{2}{|l|}{ Heavy drinking $^{\ddagger}$} \\
\hline No & $2,249(82.0)$ \\
\hline Yes & $494(18.0)$ \\
\hline Alcohol intake, drinks/wk ${ }^{\ddagger}$ & $7.20 \pm 11.97$ \\
\hline Family history of gastric cancer ${ }^{\S}$ & $446(14.7)$ \\
\hline \multicolumn{2}{|l|}{ Mucosal atrophy at baseline } \\
\hline Normal & $6,471(63.5)$ \\
\hline $\mathrm{C} 1$ & $1,018(10.0)$ \\
\hline $\mathrm{C} 2$ & $1,313(12.9)$ \\
\hline C3 & 865 (8.5) \\
\hline 01 & $338(3.3)$ \\
\hline 02 & $139(1.4)$ \\
\hline 03 & $41(0.4)$ \\
\hline \multicolumn{2}{|l|}{ Intestinal metaplasia } \\
\hline Absent & $8,759(86.0)$ \\
\hline Present & $1,426(14.0)$ \\
\hline \multicolumn{2}{|l|}{ Helicobacter pylori infection status" } \\
\hline Not infected & $3,899(40.5)$ \\
\hline Eradicated & $127(1.3)$ \\
\hline Infected & $5,597(58.2)$ \\
\hline Follow-up period, $\mathrm{yr}^{\mathrm{n}}$ & $6.88 \pm 3.18$ \\
\hline
\end{tabular}

Data are presented as mean \pm SD or number $(\%)$.

*Data available for 2,739 subjects; ${ }^{\dagger}$ Data available for 2,492 subjects;

${ }^{\ddagger}$ Data available for 2,743 subjects; ${ }^{\circledR}$ Data available for 3,041 subjects;

"Data available for 9,623 subjects; "Data available for 5,567 subjects. 
these three tests (serology, rapid urease test, or ${ }^{13} \mathrm{C}$-urea breath test) was positive or the bacteria were histologically detected by a Giemsa stain.

\section{Statistical analysis}

Descriptive statistical analyses included the calculation of rates and proportions for categorical data and of means and standard deviations for continuous data.

Associations of risk factors for AG were estimated in the 10,185 subjects by logistic regression analysis, in terms of the odds ratio (OR) and the corresponding 95\% confidence intervals (CI). Relative risks for aggravation of AG and for gastric neoplasm were evaluated in the 2,144 subjects who had undergone follow-up endoscopy using the Cox proportional hazard regression model by computing the hazard ratio (HR) and 95\% CI after multivariate adjustment.

Results were considered to be statistically significant if the two-sided p-value was less than 0.05 or if the 95\% CI did not include unity. Data analysis was performed using the SPSS version 21.0 (IBM Corp., Armonk, NY, USA).

\section{RESULTS}

\section{Baseline characteristics of the study population}

Of the 10,185 subjects (mean age, 47.8 years; male, 54.8\%), 3,714 (36.5\%) were diagnosed with AG and 1,426 (14.0\%) were diagnosed with IM. $H$. pylori was detected in 5,597 subjects (58.2\%). Of the 4,026 subjects who were $H$. pylori-negative, 127 subjects (1.3\%) had a history of $H$. pylori eradication therapy (Table 1).

\section{Associations with risk factors for AG on endoscopic finding}

Associations with risk factors for AG were estimated by logistic regression analysis. In multivariate analysis, old age ( $\geq 55$ years vs $<55$ years) (OR, 2.677; 95\% CI, 2.214 to 3.238; $\mathrm{p}<0.001)$, male sex (OR, 2.151; 95\% CI, 1.671 to 2.770; $\mathrm{p}<0.001$ ), and $H$. pylori infection (OR, 3.224; 95\% CI, 2.089 to 4.977; in eradicated vs no infection, $\mathrm{p}<0.001$; OR, 3.405; 95\% CI, 2.779 to 4.171 ; in infected vs no infection, $\mathrm{p}<0.001$ ) were significant independent risk factors for AG (Table 2).

\section{Risk factors for aggravation of AG on follow-up endoscopy}

Of the 3,714 subjects with AG, 2,144 subjects (57.7\%) had

Table 2. Associations with Risk Factors for Atrophic Gastritis Based on Endoscopic Findings

\begin{tabular}{|c|c|c|c|c|c|c|}
\hline & $\begin{array}{c}\text { AG }(-) \\
(n=6,471)\end{array}$ & $\begin{array}{c}\text { AG (+) } \\
(n=3,714)\end{array}$ & $\mathrm{OR}^{*}$ & $95 \% \mathrm{CI}$ & $\mathrm{OR}^{\dagger}$ & $95 \% \mathrm{CI}$ \\
\hline \multicolumn{7}{|l|}{ Age, yr } \\
\hline$<55$ & 5,250 & 2,089 & Reference & & Reference & \\
\hline$\geq 55$ & 1,221 & 1,625 & 3.345 & $3.057-3.659$ & 2.677 & $2.214-3.238$ \\
\hline \multicolumn{7}{|l|}{ Sex } \\
\hline Female & 3,290 & 1,314 & Reference & & Reference & \\
\hline Male & 3,181 & 2,400 & 1.889 & $1.738-2.053$ & 2.151 & $1.671-2.770$ \\
\hline \multicolumn{7}{|l|}{ Smoking $^{\ddagger}$} \\
\hline Nonsmoker & 561 & 681 & Reference & & Reference & \\
\hline Ex-smoker & 384 & 688 & 1.476 & $1.248-1.745$ & 0.854 & $0.662-1.102$ \\
\hline Current smoker & 185 & 240 & 1.069 & $0.856-1.334$ & 0.781 & $0.573-1.066$ \\
\hline \multicolumn{7}{|l|}{ Heavy drinking ${ }^{\S}$} \\
\hline No & 923 & 1,326 & Reference & & Reference & \\
\hline Yes & 191 & 303 & 1.104 & $0.904-1.348$ & 1.043 & $0.822-1.323$ \\
\hline \multicolumn{7}{|c|}{ Family history of GC" } \\
\hline No & 1,118 & 1,477 & Reference & & Reference & \\
\hline Yes & 175 & 271 & 1.172 & $0.955-1.439$ & 1.189 & $0.940-1.503$ \\
\hline \multicolumn{7}{|c|}{ H. pylori infection status ${ }^{\pi}$} \\
\hline Not infected & 3,124 & 775 & Reference & & Reference & \\
\hline Eradicated & 38 & 89 & 9.441 & $6.406-13.914$ & 3.224 & $2.089-4.977$ \\
\hline Infected & 2,890 & 2,707 & 3.776 & $3.435-4.150$ & 3.405 & $2.779-4.171$ \\
\hline
\end{tabular}

AG, atrophic gastritis; OR, odds ratio; CI, confidence interval; GC, gastric cancer; $H$. pylori, Helicobacter pylori.

*Univariate analyses of risk factors for AG; ${ }^{\dagger}$ Adjusted for age, sex, smoking, heavy drinking, family history of GC, and $H$. $p y l o r i$ infection; ${ }^{\ddagger}$ Data available for 2,739 subjects; ${ }^{8}$ Data available for 2,743 subjects; "Data available for 3,041 subjects; "Data available for 9,623 subjects. 
Table 3. Risk Factors for Aggravation of Atrophic Gastritis at Follow-up Endoscopy $(\mathrm{n}=2,144)$

\begin{tabular}{|c|c|c|c|c|c|c|}
\hline & $\begin{array}{l}\text { No change } \\
(n=1,006)\end{array}$ & $\begin{array}{c}\text { Aggravation } \\
(\mathrm{n}=1,138)\end{array}$ & $\mathrm{HR}^{*}$ & $95 \% \mathrm{CI}$ & $\mathrm{HR}^{\dagger}$ & $95 \% \mathrm{CI}$ \\
\hline \multicolumn{7}{|l|}{ Age, yr } \\
\hline$<55$ & 617 & 636 & Reference & & Reference & \\
\hline$\geq 55$ & 389 & 502 & 1.175 & $1.045-1.321$ & 1.234 & $1.065-1.430$ \\
\hline \multicolumn{7}{|l|}{ Sex } \\
\hline Female & 330 & 367 & Reference & & Reference & \\
\hline Male & 676 & 771 & 0.973 & $0.859-1.102$ & 0.900 & $0.748-1.084$ \\
\hline Smoking, pack-year ${ }^{\ddagger}$ & $11.38 \pm 15.77$ & $12.85 \pm 17.70$ & 1.001 & 0.997-1.005 & 0.999 & $0.995-1.004$ \\
\hline Alcohol intake, drinks/wk ${ }^{\S}$ & $7.23 \pm 11.65$ & $7.41 \pm 12.55$ & 1.011 & $1.006-1.017$ & 1.001 & $1.001-1.002$ \\
\hline \multicolumn{7}{|l|}{ Family history of GC" } \\
\hline No & 644 & 789 & Reference & & Reference & \\
\hline Yes & 100 & 167 & 1.050 & $0.888-1.241$ & 1.032 & $0.855-1.245$ \\
\hline \multicolumn{7}{|l|}{ H. pylori infection" } \\
\hline No & 159 & 96 & Reference & & Reference & \\
\hline Newly infected & 37 & 44 & 1.427 & $0.999-2.040$ & 1.186 & $0.788-1.785$ \\
\hline Eradicated & 177 & 143 & 1.167 & $0.901-1.512$ & 1.042 & $0.769-1.412$ \\
\hline Persistent & 437 & 685 & 1.865 & $1.506-2.310$ & 1.580 & $1.220-2.046$ \\
\hline
\end{tabular}

HR, hazard ratio; CI, confidence interval; GC, gastric cancer; H. pylori, Helicobacter pylori.

*Univariate analyses of risk factors for atrophic gastritis; ${ }^{\dagger}$ Adjusted for age, sex, smoking, alcohol intake, family history of GC, and $H$. pylori infection status; ${ }^{\ddagger}$ Data available for 1,431 subjects; ${ }^{8}$ Data available for 1,586 subjects; "Data available for 1,700 subjects; ${ }^{\circledR}$ Data available for 1,778 subjects.

undergone follow-up endoscopy. During a mean follow-up of 6.9 years (range, 1.0 to 11.2 years), 1,138 subjects (53.1\%) showed aggravation of AG and 1,006 subjects (46.9\%) showed no change. Cox proportional hazards regression revealed that age $\geq 55$ (HR, 1.234; 95\% CI, 1.065 to $1.430 ; \mathrm{p}=0.005$ ), alcohol intake (HR, 1.001; 95\% CI, 1.001 to $1.002 ; \mathrm{p}<0.001$ ), and sustained $H$. pylori infection (HR, 1.580; 95\% CI, 1.220 to 2.046; $\mathrm{p}=0.001$ ) were significantly associated with aggravation of AG (Table 3).

\section{Risk factors for gastric tumorigenesis in underlying gastric mucosal atrophy}

A total of 69 subjects was diagnosed with gastric neoplasm (32 cases of adenoma with low grade dysplasia, two cases of adenoma with high grade dysplasia, 15 cases of well-differentiated adenocarcinoma, 10 cases of moderately differentiated adenocarcinoma, four cases of poorly differentiated adenocarcinoma, and eight cases of adenocarcinoma with signet ring cell) during follow-up in AG group. The incidences of gastric neoplasm during follow-up periods were significantly different among the AG groups $(0.1 \%$ in normal, 1.6\% in $\mathrm{C} 1-\mathrm{C} 2,5.2 \%$ in $\mathrm{C} 3-01$, $12.0 \%$ in $02-03, \mathrm{p}<0.001$ ), and increased with $\mathrm{AG}$ progression $(\mathrm{r}=0.184, \mathrm{p}<0.001)$. The mean time interval to diagnosis was $6.6 \pm 2.7$ years in mild AG $(21 / 1,324,1.6 \%), 5.8 \pm 3.4$ years in moderate AG $(37 / 670,5.2 \%)$, and $4.9 \pm 2.4$ years in severe AG $(11 / 81,12.0 \%)(\mathrm{r}=-0.188, \mathrm{p}=0.035)$. The independent risk fac- tors for gastric neoplasm in underlying mucosal atrophy were age $\geq 55$ (HR, 2.582; 95\% CI, 1.375 to 4.849; $\mathrm{p}=0.003$ ), alcohol intake (HR, 1.003; 95\% CI, 1.001 to $1.004 ; \mathrm{p}<0.001$ ), baseline AG (HR, 2.285; 95\% CI, 1.141 to 4.576; in moderate vs mild, $\mathrm{p}=0.020$; HR, 4.187; 95\% CI, 1.609 to 10.896 ; in severe vs mild, $\mathrm{p}=0.003$ ), and presence of IM (HR, 2.655; 95\% CI, 1.306 to 5.398; $\mathrm{p}=0.007$ ). However, $H$. pylori infection was not significantly associated with the development of gastric neoplasm (Table 4).

\section{DISCUSSION}

In this study, we analyzed the risk factors for the aggravation of AG and gastric tumorigenesis in underlying gastric mucosal atrophy. Moderate to severe AG and presence of IM were significant risk factors for gastric neoplasm. Although $H$. pylori infection was a risk factor for aggravating gastric mucosal atrophy, it was not significantly associated with the development of gastric neoplasm. It could be explained by the concepts of "point of no return" in the histological cascade from chronic gastritis to cancer. ${ }^{16} \mathrm{H}$. pylori has been believed to influence on gastric carcinogenesis through the development of AG. ${ }^{17}$ A nationwide cohort study which has reported from Taiwan found that a cohort of patients that underwent early $H$. pylori eradication exhibited a similar risk of gastric cancer compared with the general population, whereas the cohort of patients that underwent late eradication exhibited a higher risk of gastric cancer compared to the 
Table 4. Risk Factors for Gastric Tumorigenesis in Underlying Gastric Mucosal Atrophy $(n=2,144)$

\begin{tabular}{|c|c|c|c|c|c|c|}
\hline & $\begin{array}{c}\text { Control } \\
(\mathrm{n}=2,075)\end{array}$ & $\begin{array}{c}\text { Neoplasm } \\
(\mathrm{n}=69)\end{array}$ & $\mathrm{HR}^{*}$ & $95 \% \mathrm{CI}$ & $\mathrm{HR}^{\dagger}$ & $95 \% \mathrm{CI}$ \\
\hline \multicolumn{7}{|l|}{ Age, yr } \\
\hline$<55$ & 1,225 & 28 & Reference & & Reference & \\
\hline$\geq 55$ & 850 & 41 & 2.134 & $1.319-3.451$ & 2.582 & $1.375-4.849$ \\
\hline \multicolumn{7}{|l|}{ Sex } \\
\hline Female & 687 & 10 & Reference & & Reference & \\
\hline Male & 1,388 & 59 & 2.790 & $1.427-5.456$ & 1.766 & $0.656-4.752$ \\
\hline Smoking, pack-year ${ }^{\ddagger}$ & $11.90 \pm 16.75$ & $21.38 \pm 19.16$ & 1.020 & $1.009-1.031$ & 1.006 & $0.993-1.019$ \\
\hline Alcohol intake, drinks/wk ${ }^{\S}$ & $7.13 \pm 12.00$ & $13.18 \pm 14.96$ & 1.029 & $1.016-1.042$ & 1.003 & $1.001-1.004$ \\
\hline \multicolumn{7}{|l|}{ Family history of GC" } \\
\hline No & 1,389 & 44 & Reference & & Reference & \\
\hline Yes & 254 & 13 & 1.444 & $0.777-2.683$ & 1.405 & $0.712-2.771$ \\
\hline \multicolumn{7}{|l|}{ H. pylori infection" } \\
\hline No & 245 & 10 & Reference & & Reference & \\
\hline Newly infected & 78 & 3 & 0.923 & $0.254-3.355$ & 0.482 & $0.100-2.313$ \\
\hline Eradicated & 302 & 18 & 1.407 & $0.650-3.049$ & 1.057 & $0.444-2.518$ \\
\hline Persistent & 1,094 & 28 & 0.706 & $0.343-1.454$ & 0.492 & $0.211-1.147$ \\
\hline \multicolumn{7}{|l|}{ Baseline AG } \\
\hline Mild (C1, C2) & 1,324 & 21 & Reference & & Reference & \\
\hline Moderate $(\mathrm{C} 3,01)$ & 670 & 37 & 3.471 & $2.032-5.930$ & 2.285 & $1.141-4.576$ \\
\hline Severe $(02,03)$ & 81 & 11 & 9.496 & $4.575-19.711$ & 4.187 & $1.609-10.896$ \\
\hline \multicolumn{7}{|l|}{ Intestinal metaplasia } \\
\hline Absent & 1,266 & 17 & Reference & & Reference & \\
\hline Present & 809 & 52 & 4.689 & $2.712-8.109$ & 2.655 & $1.306-5.398$ \\
\hline
\end{tabular}

HR, hazard ratio; CI, confidence interval; GC, gastric cancer; H. pylori, Helicobacter pylori; AG, atrophic gastritis.

*Univariate analyses of risk factors for gastric neoplasms; ${ }^{\dagger}$ Adjusted for age, sex, smoking, alcohol intake, family history of GC, $H$. pylori infection, baseline AG, and intestinal metaplasia; ${ }^{\ddagger}$ Data available for 1,431 subjects; ${ }^{8}$ Data available for 1,586 subjects; "Data available for 1,700 subjects; "Data available for 1,778 subjects.

general population. ${ }^{18}$ In a Chinese randomized trial with 1,630 $\mathrm{H}$. pylori-infected subjects who were followed up for 7.5 years, $H$. pylori eradication had failed to reduce the incidence of gastric cancer, but in subgroup analysis of patients without precancerous lesions such as AG, IM, or dysplasia, H. pylori eradication showed risk reduction of gastric cancer development. ${ }^{19}$ A recent meta-analysis also demonstrated that $H$. pylori eradication was not beneficial in patients with IM or dysplasia regarding a reduction of risk of gastric cancer. ${ }^{16}$

Long-standing $H$. pylori infection would be an important risk factor for the aggravation of gastric mucosal atrophy. However, H. pylori might not increase the risk of gastric neoplasm after AG had been already developed, even though it had an important role in gastric carcinogenesis.

AG is usually diagnosed by histologic examination of gastric mucosa. However, multiple biopsies can be invasive and timeconsuming procedures, and a few endoscopic biopsy samples may not reflect the entire extent of atrophy. ${ }^{20}$ Although endoscopic diagnosis of AG is a basic method, it is flawed by a low sensitivity, specificity and inter-observer variation. ${ }^{21}$ A recent study identified significant correlations between endoscopic and histological diagnosis of AG. ${ }^{20}$ Serum pepsinogen test is known to be another method for the diagnosis of AG. Measurement of the levels of serum pepsinogen may help detect AG or IM in a noninvasive manner, but its efficiency remains controversial. ${ }^{22}$ The value of pepsinogen level as a biomarker of AG could vary among countries or populations, and may be affected by gastric acid inhibitors or some medical conditions. ${ }^{20}$ Therefore, the serum pepsinogen test would be more suitable for mass screening of gastric cancer for the high-risk population. ${ }^{23}$ Even though the endoscopic diagnosis of AG may have some limitations, we adopted this method to assess AG because most of the patients were followed up annually with endoscopy for cancer screening. In this study, AG and IM were evaluated by reviewing endoscopic images, and several meetings were held to improve the accuracy of diagnosis and minimize inter-observer variation by extensively reviewing multiple cases with AG. Inter-observer agreement of atrophy was low initially $(\kappa=0.36, p<0.001)$ and 
improved gradually but substantially $(\kappa=0.62, \mathrm{p}<0.001)$.

AG has been regarded as a precancerous lesion, but many patients with AG rarely experience gastric cancer during their lifetime. In this study, we focused on the process of developing gastric neoplasm (adenoma and cancer) from AG and the risk factors for gastric neoplasm in underlying gastric mucosal atrophy. In the results, old age, alcohol intake, extent of AG, and presence of IM were significantly associated with gastric neoplasm. In contrast, sex, smoking and a family history of gastric cancer was not associated with gastric neoplasm in AG. Male sex was a risk factor for AG but not for the aggravation of AG or the development of gastric neoplasm. These results are similar to the previous large-scaled observational study which reported that male sex was an independent risk factor for gastric precancerous conditions but not for gastric cancer. ${ }^{24}$ These findings imply that male sex may be an increased risk of gastric cancer, especially in the early process of carcinogenesis such as AG. The mechanism of the sex-related differences in gastric carcinogenesis is still unknown, and further studies will be needed.

A possible relationship between alcohol consumption and risk for gastric cancer has long been hypothesized, but epidemiological evidence has been inconclusive. ${ }^{25}$ The present study demonstrated that alcohol intake resulted in an increased risk for aggravation of AG and for gastric neoplasm.

The strength of this study includes a large number of subjects and long-term follow-up data. To the best of our knowledge, this study is the largest one that evaluates risk factors for gastric neoplasm in underlying mucosal atrophy. Moreover, the study population can be a representative of the general population because many of the subjects were asymptomatic and underwent endoscopy as part of a health check-up.

This study has some limitations. First, the rate of follow-up loss was approximately $40 \%$ of subjects who were diagnosed with AG during baseline endoscopy, which could represent a selection bias in this retrospective cohort study. Second, as the extent of AG was evaluated by spot endoscopic image, it might be underestimated by insufficient endoscopic images. To decrease this underestimation, we had reviewed both images and descriptions of each endoscopic examination.

In conclusion, extensive mucosal atrophy and presence of IM increase the risk for gastric neoplasm in underlying mucosal atrophy. Alcohol consumption is also a risk factor for gastric neoplasm. Therefore, annual endoscopic surveillance may be appropriate to screen gastric neoplasm in high-risk individuals who consume alcohol as well as exhibit moderate to severe mucosal atrophy and IM. Further prospective studies are needed to evaluate the role of endoscopic surveillance in high-risk individuals with $\mathrm{AG}$.

\section{CONFLICTS OF INTEREST}

No potential conflict of interest relevant to this article was reported.

\section{ACKNOWLEDGEMENTS}

This work was supported by Promising-Pioneering Researcher Program through Seoul National University in 2015.

\section{REFERENCES}

1. Correa P. Human gastric carcinogenesis: a multistep and multifactorial process--first American Cancer Society award lecture on cancer epidemiology and prevention. Cancer Res 1992;52:67356740.

2. Watari J, Chen N, Amenta PS, et al. Helicobacter pylori associated chronic gastritis, clinical syndromes, precancerous lesions, and pathogenesis of gastric cancer development. World J Gastroenterol 2014;20:5461-5473.

3. de Vries AC, Haringsma J, Kuipers EJ. The detection, surveillance and treatment of premalignant gastric lesions related to Helicobacter pylori infection. Helicobacter 2007;12:1-15.

4. Karimi P, Islami F, Anandasabapathy S, Freedman ND, Kamangar F. Gastric cancer: descriptive epidemiology, risk factors, screening, and prevention. Cancer Epidemiol Biomarkers Prev 2014;23:700713.

5. Ferlay J, Shin HR, Bray F, Forman D, Mathers C, Parkin DM. Estimates of worldwide burden of cancer in 2008: GLOBOCAN 2008. Int J Cancer 2010;127:2893-2917.

6. Forman D, Burley VJ. Gastric cancer: global pattern of the disease and an overview of environmental risk factors. Best Pract Res Clin Gastroenterol 2006;20:633-649.

7. Anderson WF, Camargo MC, Fraumeni JF Jr, Correa P, Rosenberg PS, Rabkin CS. Age-specific trends in incidence of noncardia gastric cancer in US adults. JAMA 2010;303:1723-1728.

8. Song H, Held M, Sandin S, et al. Increase in the prevalence of atrophic gastritis among adults age 35 to 44 years old in Northern Sweden between 1990 and 2009. Clin Gastroenterol Hepatol 2015;13:1592-1600.e1.

9. Song H, Ekheden IG, Zheng Z, Ericsson J, Nyrén O, Ye W. Incidence of gastric cancer among patients with gastric precancerous lesions: observational cohort study in a low risk Western population. BMJ 2015;351:h3867.

10. Joo YE, Park HK, Myung DS, et al. Prevalence and risk factors of atrophic gastritis and intestinal metaplasia: a nationwide multicenter prospective study in Korea. Gut Liver 2013;7:303-310.

11. Lee KS, Oh DK, Han MA, et al. Gastric cancer screening in Korea: report on the national cancer screening program in 2008. Cancer Res Treat 2011;43:83-88.

12. Chung SJ, Park MJ, Kang SJ, et al. Effect of annual endoscopic screening on clinicopathologic characteristics and treatment modality of gastric cancer in a high-incidence region of Korea. Int $\mathrm{J}$ Cancer 2012;131:2376-2384

13. Yoon H, Kim N, Lee HS, et al. Effect of endoscopic screening at 
1-year intervals on the clinicopathologic characteristics and treatment of gastric cancer in South Korea. J Gastroenterol Hepatol 2012;27:928-934.

14. Kimura K, Takemoto T. An endoscopic recognition of the atrophic border and its significance in chronic gastritis. Endoscopy 1969;3:87-97.

15. Masuyama H, Yoshitake N, Sasai T, et al. Relationship between the degree of endoscopic atrophy of the gastric mucosa and carcinogenic risk. Digestion 2015;91:30-36.

16. Chen HN, Wang Z, Li X, Zhou ZG. Helicobacter pylori eradication cannot reduce the risk of gastric cancer in patients with intestinal metaplasia and dysplasia: evidence from a meta-analysis. Gastric Cancer 2016;19:166-175.

17. Ohata H, Kitauchi S, Yoshimura N, et al. Progression of chronic atrophic gastritis associated with Helicobacter pylori infection increases risk of gastric cancer. Int J Cancer 2004;109:138-143.

18. Wu CY, Kuo KN, Wu MS, Chen YJ, Wang CB, Lin JT. Early Helicobacter pylori eradication decreases risk of gastric cancer in patients with peptic ulcer disease. Gastroenterology 2009;137:16411648.e2.

19. Wong BC, Lam SK, Wong WM, et al. Helicobacter pylori eradication to prevent gastric cancer in a high-risk region of China: a randomized controlled trial. JAMA 2004;291:187-194.

20. Lee JY, Kim N, Lee HS, et al. Correlations among endoscopic, histologic and serologic diagnoses for the assessment of atrophic gastritis. J Cancer Prev 2014;19:47-55.

21. Park YH, Kim N. Review of atrophic gastritis and intestinal metaplasia as a premalignant lesion of gastric cancer. J Cancer Prev 2015;20:25-40.

22. Huang YK, Yu JC, Kang WM, et al. Significance of serum pepsinogens as a biomarker for gastric cancer and atrophic gastritis screening: a systematic review and meta-analysis. PLoS One 2015;10:e0142080.

23. Kim EH, Kang H, Park CH, et al. The optimal serum pepsinogen cut-off value for predicting histologically confirmed atrophic gastritis. Dig Liver Dis 2015;47:663-668.

24. Park YM, Kim JH, Baik SJ, Park JJ, Youn YH, Park H. Clinical risk assessment for gastric cancer in asymptomatic population after a health check-up: an individualized consideration of the risk factors. Medicine (Baltimore) 2016;95:e5351.

25. IARC Working Group on the Evaluation of Carcinogenic Risks to Humans. Alcohol consumption and ethyl carbamate. IARC Monogr Eval Carcinog Risks Hum 2010;96:3-1383. 